\title{
66. Studies on Pillaromycin, a New Antibiotic
}

\author{
By Motoo Shibata, Mitsuko Asai, Kōmei Mizuno, \\ Akira MiYaKe, and Sueo TATSUOKA \\ Research Laboratories, Takeda Chemical Industries, Ltd.*) \\ (Comm. by Yasuhiko AsAhinA, M.J.A., April 13, 1964)
}

Pillaromycin is a group consisting of new anti-acidfast bacterial and anti-tumor antibiotics and is produced by Streptomyces flavovirens No. 65786. It is separated into $A, B_{I}, B_{I I}$, and $C$ and among these components pillaromycin A (abbr. PMA) is the main component and the strongest in anti-bacterial and anti-tumor activities.

PMA crystallizes from ethyl acetate in the form of yellow prisms, m. p. $208^{\circ},[\alpha]_{\mathrm{D}}^{20}=-33^{\circ}(\mathrm{MeOH})$. The elementary analysis and the molecular weight measured by the osmotic pressure method indicated that PMA has the formula of $\mathrm{C}_{25} \mathrm{H}_{26} \mathrm{O}_{10}$. It is soluble in many polar organic solvents and aqueous sodium hydrogen carbonate and is positive to the Fehling reagent. It gives a blue color with Barton's reagent $\left(\mathrm{FeCl}_{3} \cdot \mathrm{K}_{3} \mathrm{Fe}(\mathrm{CN})_{6}\right)$, a violet-red color with concentrated sulfuric acid, but is negative to magnesium acetate reaction. The UV spectrum of PMA indicates the presence of an $\alpha, \alpha^{\prime}$-dihydroxyanthraquinone or a flavoskyrin-like structure and the IR spectrum indicates the presence of three kinds of carbonyl groups: an isolated carbonyl at $1720 \mathrm{~cm}^{-1}$, a conjugated carbonyl at $1685 \mathrm{~cm}^{-1}$, and a chelated carbonyl at $1630 \mathrm{~cm}^{-1}$. The $\mathrm{Nmr}$ studies of PMA and a pentaacetylPMA indicate the presence of a $>\mathrm{CH}-\mathrm{CH}_{3}$ group (at $0.85 \mathrm{ppm}$, doublet) and a $-\mathrm{CH}_{3}$ group (at $2.4 \mathrm{ppm}$, singlet) and an acidic or weakly hydrogen bonding proton (at lower magnetic field).

PMA is degradated quantitatively by heating with $50 \%$ aqueous acetic acid into a water soluble portion (pillarose, $\mathrm{C}_{5} \mathrm{H}_{10} \mathrm{O}_{3}$; (II)) and a lipophylic portion (pillaromycinone (abbr. $\mathrm{PMN}$ ), $\mathrm{C}_{20} \mathrm{H}_{10} \mathrm{O}_{7}$; (III)). II gives a mono-2, 4-dinitrophenylhydrazone and from its elementary analysis the molecular formula, $\mathrm{C}_{5} \mathrm{H}_{10} \mathrm{O}_{3}$, is given to II. The $\mathrm{Nmr}$ studies of II indicates the presence of a $>\mathrm{CH}-\mathrm{CH}_{3}$ group. II gives a blue-violet color characteristic of 2-deoxy-pentose with acetic acid. benzidine. The presence of a $-\mathrm{CH}(\mathrm{OH})-\mathrm{CH}_{3}$ group is shown by the iodoform reaction. Lead tetraacetate oxidation of II gives 2 moles of acetaldehyde. It follows that II can be represented by the 2,5dideoxypentose (II). Upon mild $50 \%$ aqueous acetic acid degradation of pentaacetyl-PMA a tetraacetate is obtained, which is proved to be identical with the tetraacetate of PMN obtained directly by acetyla-

\footnotetext{
*) Juso-Nishino-cho, Higashiyodogawa-ku, Osaka.
} 
tion of PMN. This result indicates that the position of attachment of II to PMN can not be on any of the four hydroxyls which are already acetylated in pentaacetyl-PMA. The calculation of molecular formulae of I, II, and III and the absence of an isolated carbonyl peak in the IR spectrum of PMN indicate that elimination rather than hydrolysis occurred in this reaction. When the UV spectrum of PMN is compared with that of PMA, no shift is observed of the absorption maximum. The Nmr studies of PMN and tetraacetyl-PMN indicate the presence of two aliphatic hydroxyls, two aromatic hydroxyls and a $-\mathrm{CH}_{3}$ group (at $2.4 \mathrm{ppm}$, singlet).

Treatment of PMN with dimethyl sulfate and potassium carbonate in acetone gives a di-O-methyl ether. This compound is oxidized by alkaline permanganate to give a mixture of acidic fragments. They are separated into oxalic acid, 3-methoxy-1,2,4,5-benzenetetracarboxylic acid and 3-methoxyphthalic acid, all identical with the authentic samples. It is also confirmed that by this alkaline permanganate oxidation 3-methoxy-1, 2, 4, 5-benzenetetracarboxylic acid does not give 3-methoxy phthalic acid. From these facts the partial structure, (a) or (b) is given to PMN.

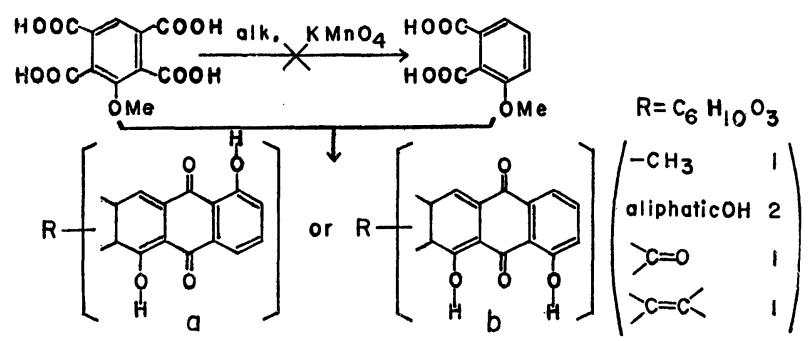

PMN gives a mono-thiosemicarbazone and a mono-2, 4-dinitrophenylhydrazone. The UV spectra of these compounds indicate the presence of an $\alpha$, $\beta$-conjugated carbonyl which is isolated from other chromophores in PMN. Catalytic reduction of PMN on Adams' platinum gives a dihydro- $\mathrm{PMN}\left(\mathrm{C}_{20} \mathrm{H}_{18} \mathrm{O}_{7}\right)$. Further reduction of dihydroPMN on palladium-carbon gives rise to removal of two oxygen atoms in aliphatic hydroxyl groups and a compound $\left(\mathrm{C}_{20} \mathrm{H}_{18} \mathrm{O}_{5}\right)$, which is referred to as pillaronone, is obtained. It is also derived directly from PMN by catalytic reduction on palladium-carbon. The UV spectrum of pillaronone is similar to that of PMN and it follows that during the course of reduction no drastic change in the chromophore moiety of PMN was occurred. Pillaronone also gives a mono-2, 4-dinitrophenylhydrazone. The UV spectrum of this compound indicates the presence of an isolated carbonyl. The IR spectrum of pillaronone indicates the presence of hydrogen bonding carbonyl and 
the absence of an isolated and a conjugated carbonyls. Acetylation of pillaronone with hot acetic anhydride-pyridine gives a diacetylpillaronone. The Nmr studies of this compound indicate the presence of two aromatic hydroxyls and the absence of an aliphatic hydroxyl in pillaronone. The signals of $-\mathrm{CH}_{3}$ groups in PMA, PMN, dihydroPMN and pillaronone-mono-2, 4-DNPH appear at $2.1 \sim 2.4 \mathrm{ppm}$, on the other hand those in pillaronone and diacetyl-pillaronone appear at 1.7 ppm. The Nmr spectrum of diacetyl-pillaronone measured in $\mathrm{CDCl}_{3}$ indicates the presence of a tert. hydroxyl (at $4.4 \mathrm{ppm}$ ) which disappears when exchanged with $\mathrm{D}_{2} \mathrm{O}$. These facts are well explained by the following mechanism, namely the $-\mathrm{CH}_{3}$ group in question exists as $\mathrm{CO}-\mathrm{CH}_{3}$ in $\mathrm{PMN}$ and converts on catalytic reduction to hemi-ketal configuration (in pillaronone) and returns with strong acid to $\mathrm{CO}-\mathrm{CH}_{3}$ configuration (in pillaronone-mono-2, 4-DNPH). Combining hitherto obtained findings, a partial structure (c) is derived for $-\mathrm{CH}_{3},>\mathrm{C}=\mathrm{O}$, and $>\mathrm{C}=\mathrm{C}<$ groups in the $\mathrm{R}$ portion of $\mathrm{PMN}$.

Next, two aliphatic hydroxyls in the $R$ portion of PMN are investigated. The $\mathrm{Nmr}$ studies of tetraacetyl-PMN indicated the presence of two aliphatic hydroxyls and this fact is also confirmed by the formation of a ditosylate of PMN. Oxidation of PMN and its derivatives with various oxidants such as lead tetraacetate, hydrogen peroxide and periodic acid indicates the absence of primary hydroxyl and reveals the relative position of the two hydroxyls in PMN that they exist vicinally in a ring system. The formation of PMN-acetonide supports this conclusion. $\mathrm{A}-\mathrm{CH}_{3},>\mathrm{C}=\mathrm{O},>\mathrm{C}=\mathrm{C}<$, two hydroxyls and a ring skeleton in the $\mathrm{R}$ portion of $\mathrm{PMN}$ are assigned as schemata (d) or (e).
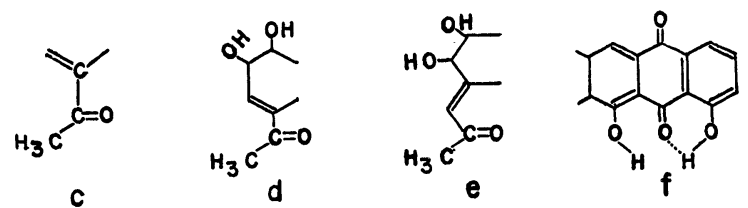

The findings hitherto obtained indicate that combination of partial structures (a) or (b) with (d) or (e) and addition of one mole of hydrogen to partial structure (a) or (b) make the complete structure of PMN. In this case it is important to recall that PMA and PMN give no color reaction with methanolic magnesium acetate and are both soluble in aqueous sodium hydrogen carbonate and the value of optical rotation of $\mathrm{PMN}$ is $+550^{\circ}$. These properties are in good agreement with these of skyrin group compounds which were reported by Raistrick ${ }^{13}$ and Shibata. ${ }^{2,3)}$ Treatment of PMN-di-tosylate in ethanol with sodium benzylmercaptan gives a PMN-di-tosyl-mono-benzyl 
mercaptal. This compound is further treated with ethanedithiol and boron trifluoride to give a mono-tosyl-mono-benzyl-mercapto-di-ethylenethioketal. The Nmr spectra and IR spectra of these two derivatives and PMA and PMN-acetonide support the partial structure (f) which has closer relation to (b) rather than to (a). Taking account into the fact that hemi-ketal formation takes place in the $\mathrm{R}$ portion, schema (f) is combined with (d) and (e) to give schemata (g) and (h). The fact that cautious zine dust distillation of $\mathrm{PMN}$ gives anthracene and naphthacene also supports the schema (g).
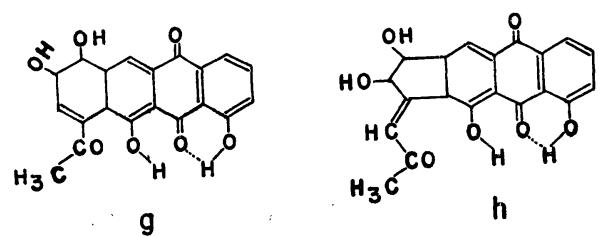

As mentioned above the position of attachment of pillarose is assigned to the $\beta$-position of $\mathrm{CO}-\mathrm{CH}_{3}$ of $\mathrm{PMN}$. This assignment satisfies the fact that upon degradation with $50 \%$ acetic acid PMA underwent $\beta$-elimination to give an $\alpha, \beta$-conjugated carbonyl.

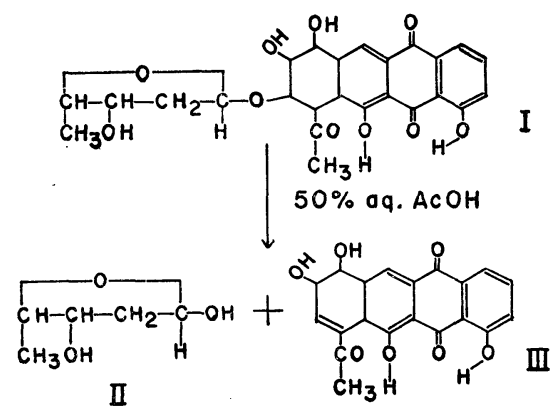

We wish to acknowlege Professors S. Shibata and K. Nakanishi, and Drs. K. Tanaka and K. Nakazawa for their unfailing interest and encouragement.

\section{References}

1) Breen, J., J. C. Dacre, H. Raistrick, and G. Smith: Studies in the biochemistry of micro-organisms 95. Rugulosin, a crystalline colouring matter of Penicillium rugulosum Thom. Biochem. J., 60, 618 (1955).

2) Shibata, S., T. Murakami, and M. Takido: Metabolic products of fungi IX. Rugulosin (2). The structure of rugulosin and its relation to the structure of flavoskyrin. Pharm. Bull. (Tokyo), 4, 303 (1956).

3) Shibata, S., T. Ikekawa, and T. Kishi: Metabolic products of fungi XVII. The structure of flavoskyrin (2). Chem. Pharm. Bull. (Tokyo), 8, 889 (1960). 